\title{
AN EFFICIENT COMPUTATIONAL APPROACH TO A CLASS OF MINMAX OPTIMAL CONTROL PROBLEMS WITH APPLICATIONS
}

\author{
B. $\mathrm{LI}^{凶 1,2}$, K. L. TEO ${ }^{1,2}$, G. H. ZHAO 3 and G. R. DUAN ${ }^{1}$
}

(Received 31 August, 2009; revised 6 February, 2010)

\begin{abstract}
In this paper, an efficient computation method is developed for solving a general class of minmax optimal control problems, where the minimum deviation from the violation of the continuous state inequality constraints is maximized. The constraint transcription method is used to construct a smooth approximate function for each of the continuous state inequality constraints. We then obtain an approximate optimal control problem with the integral of the summation of these smooth approximate functions as its cost function. A necessary condition and a sufficient condition are derived showing the relationship between the original problem and the smooth approximate problem. We then construct a violation function from the solution of the smooth approximate optimal control problem and the original continuous state inequality constraints in such a way that the optimal control of the minmax problem is equivalent to the largest root of the violation function, and hence can be solved by the bisection search method. The control parametrization and a time scaling transform are applied to these optimal control problems. We then consider two practical problems: the obstacle avoidance optimal control problem and the abort landing of an aircraft in a windshear downburst.
\end{abstract}

2000 Mathematics subject classification: primary 49J15.

Keywords and phrases: minmax optimal control, continuous state inequality constraints, constraint transcription, computation method, control parametrization, time scaling transform, root finding, obstacle avoidance, aircraft abort landing, windshear downburst.

\section{Introduction}

Robot navigation problems have been extensively studied in the literature. One approach, known as the reactive approach (see [1]), is to design a specific control law for each behaviour within a collection of behaviours, dedicated to performing a specific

\footnotetext{
${ }^{1}$ Harbin Institute of Technology, PR China; e-mail: g.r.duan@hit.edu.cn.

${ }^{2}$ Curtin University of Technology, Australia; e-mail: bin.li@postgrad.curtin.edu.au,

k.1.teo@curtin.edu.au.

${ }^{3}$ Dalian University of Technology, PR China; e-mail: ghzhao6961@hotmail.com.

(C) Australian Mathematical Society 2010, Serial-fee code 1446-1811/2010\$16.00
} 
task. The robot switches between different behaviours when different circumstances are encountered in the environment.

In [2], the problem of a single robot moving towards a goal while avoiding obstacles is considered, where the optimal sequence of switches between the go-to-goal mode and the avoid-obstacle mode is to be found. This optimization problem is solved as an optimal control problem. The radius of the obstacle, called the safety distance, is regarded as the control parameter. However, when the robot crosses the boundary of the safety region known as the guard, it changes its mode. But then it may be steered back towards the guard, thus traversing the guard many times in a short time interval. To avoid this chattering situation, the single guard is replaced in [3] by two circles with a common centre at the obstacle. When the robot is in the goal-approach mode and is outside the inner circle, it will change its mode when the inner circle is crossed. On the other hand, when the robot is in the avoid-obstacle mode and is inside the outer circle, it will change its mode when it traverses towards the outer circle. The radii are regarded as control parameters. The optimal radii are obtained by solving an optimal control problem using a gradient-based method. However, neither of the guard or two circle approaches solves this obstacle avoidance problem optimally.

A low-altitude windshear is a meteorological phenomenon most common in subtropical regions. It is always associated with high ground temperatures leading to a "downburst", that is, air descends at high speed and then spreads out horizontally, still at high speed. It is highly dangerous when an airplane encounters such a windshear, even for a highly skilled pilot. This is because the aircraft may encounter a headwind followed by a tailwind, both coupled with a downdraft. The transition from the headwind to the tailwind leads to an acceleration and the resulting windshear inertial force can be as large as the drag of the aircraft, and sometimes as large as the thrust of the engines. Due to several fatal accidents caused by windshears, the abort landing problem has been extensively studied in the literature (see, for example, $[4,5,8]$ ). This problem can be formulated as a minmax optimal control problem. The problem is solved numerically in [8] by the gradient-restoration method, and in [4, 5] by the multiple shooting method, which requires a good guess at the initial condition. This is not an easy task to achieve.

In this paper, we derive an efficient computational method for solving a general minmax optimal control problem, where the minimum deviation from the violation of the continuous state inequality constraints is maximized. The constraint transcription method [6] is used to construct a smooth approximate function for each of the continuous state inequality constraints. We then obtain a smooth approximate optimal control problem with the integral of the summation of these smooth approximate functions as its cost function. A necessary condition and a sufficient condition are derived showing the relationship between the original problem and this smooth approximate problem. We then construct a violation function from the solution of the smooth approximate optimal control problem and the original continuous state inequality constraints in such a way that the problem of finding an optimal control of the minmax optimal control problem is equivalent to the problem of finding the 
largest root of the violation function. The control parametrization technique [9] and a time scaling transform [10] are applied to these optimal control problems. In [11], a root finding algorithm is proposed for the smooth approximate function. However, its largest root does not correspond to the optimal solution of the minmax optimal control problem.

We show that the two practical problems (that is, the obstacle avoidance problem of an autonomous mobile robot and the abort landing of an aircraft in a windshear downburst) discussed above can be formulated as special cases of the general minmax optimal control problem. The proposed computational method is applied to solve these problems. The solutions obtained are highly satisfactory.

\section{Problem statement}

Consider a dynamical system defined on $[0, T]$,

$$
\begin{gathered}
\dot{x}(t)=f(x(t), u(t)), \quad t \in(0, T], \\
x(0)=x^{0}
\end{gathered}
$$

with terminal condition

$$
x(T)=x^{f}
$$

where $T$ is the terminal time, $x=\left[x_{1}, \ldots, x_{n}\right]^{\top} \in \mathbb{R}^{n}$ is the state vector, $u=$ $\left[u_{1}, \ldots u_{r}\right]^{\top} \in \mathbb{R}^{r}$ the control vector while $f=\left[f_{1}, \ldots, f_{n}\right]^{\top} \in \mathbb{R}^{n}$ is a given continuously differentiable function of its arguments.

We assume that the following condition is satisfied.

(A1) Let $\mathbb{V}$ be a compact subset of $\mathbb{R}^{r}$. Then, there exists a constant $K_{1}$ such that

$$
|f(x, u)| \leq K_{1}(1+|x|) \quad \forall(x, u) \in \mathbb{R}^{n} \times \mathbb{V},
$$

where $|\cdot|$ denotes the Euclidean norm.

Define

$$
U=\left\{v=\left[v_{1}, \ldots v_{r}\right]^{\top} \in \mathbb{R}^{r}: \alpha_{i} \leq v_{i} \leq \beta_{i}, i=1, \ldots, r\right\}
$$

where $\alpha_{i}$ and $\beta_{i}(i=1, \ldots, r)$ are given real numbers. A piecewise continuous function $u$ is said to be an admissible control if $u(t) \in U$ for all $t \in[0, T]$. Let $\mathcal{U}$ be the class of all such admissible controls. Furthermore, let $x(\cdot \mid u)$ denote the solution of System (2.1) corresponding to $u \in \mathcal{U}$.

Consider the continuous state inequality constraints

$$
g_{i}(x(t \mid u), \delta) \geq 0, \quad \forall t \in[0, T], i=1, \ldots, N .
$$

It is assumed that the following conditions are satisfied.

(A2) For each $i=1, \ldots, N, g_{i}(x, \delta)$ is a strictly monotonically decreasing function as a function of $\delta$ for $\delta \geq 0$. 
(A3) For each $u \in \mathcal{U}$, there exists $\delta^{u}>0$ such that

$$
\min _{\substack{1 \leq i \leq N \\ 0 \leq t \leq T}} g_{i}\left(x(t \mid u), \delta^{u}\right)=0 .
$$

(A4) $g_{i}, i=1, \ldots, N$, are continuously differentiable with respect to $x$ and $\delta$.

We may now state our minmax optimal control problem as follows.

PRoBlem P. Given the dynamical system (2.1) subject to the terminal constraint (2.2) and the continuous state inequality constraints (2.3), find a control $u \in \mathcal{U}$ such that $\delta$ is maximized.

In Problem $\mathrm{P}, \delta$ is called the violation parameter. It is the size parameter in the obstacle-avoidance problem and the height of the aircraft in the abort landing problem.

REMARK 1. By (A1) and the definition of $\mathcal{U}$, it follows from an argument similar to that given in [9, Proof of Lemma 6.4.2] that $x(t \mid u) \in X$ for all $t \in[0, T]$ and for all $u \in \mathcal{U}$, where $X \subset \mathbb{R}^{n}$ is a compact subset. Thus, by (A2)-(A4), it can be shown that there exists a $\widehat{\delta}, 0 \leq \widehat{\delta}<\infty$, such that $\delta^{u} \leq \widehat{\delta}$ for all $u \in \mathcal{U}$.

\section{Computation method}

To solve Problem P, we shall apply the control parametrization scheme [9] together with a time scaling transform [10]. The time horizon $[0, T]$ is partitioned by a monotonically increasing sequence $\left\{t_{0}, \ldots, t_{p}\right\}$. Then, the control is approximated by a piecewise constant function

$$
u^{p}(t)=\sum_{i=1}^{p} \sigma^{i} \chi_{\left[t_{i-1}, t_{i}\right)}(t),
$$

where $t_{i-1} \leq t_{i}, i=1, \ldots, p$, with $t_{0}=0$ and $t_{p}=T$, and

$$
\chi_{I}(t)= \begin{cases}1 & \text { if } t \in I, \\ 0 & \text { otherwise }\end{cases}
$$

As $u^{p} \in \mathcal{U}, \sigma^{i}=\left[\sigma_{1}^{i}, \ldots, \sigma_{r}^{i}\right]^{\top} \in U$ for $i=1, \ldots, p$. Denote by $\Xi$ the set of all such $\sigma=\left[\left(\sigma^{1}\right)^{\top}, \ldots,\left(\sigma^{p}\right)^{\top}\right]^{\top} \in \mathbb{R}^{p r}$.

The switching times $t_{i}, 1 \leq i \leq p-1$, are regarded as additional decision variables. We shall employ the time scaling transform introduced in [10] to map these switching times into a set of fixed time points $k / p, k=1, \ldots, p-1$, on a new time horizon $[0,1]$. This is easily achieved by the differential equation

$$
\frac{d t(s)}{d s}=v^{p}(s), \quad s \in[0,1],
$$


with initial condition

$$
t(0)=0
$$

where

$$
v^{p}(s)=\sum_{i=1}^{p} \theta_{i} \chi_{\left[\frac{i-1}{p}, \frac{i}{p}\right)}(s)
$$

Here $\theta_{i} \geq 0, i=1, \ldots, p$, and

$$
\sum_{i=1}^{p} \frac{\theta_{i}}{p}=T
$$

Let $\theta=\left[\theta_{1}, \ldots, \theta_{p}\right]^{\top}$ and let $\Theta$ be the set containing all such $\theta$.

Integrating (3.2) with initial condition (3.3), it is easy to see that

$$
t(s)=\sum_{k=1}^{q-1} \frac{\theta_{k}}{p}+\frac{\theta_{q}}{p}(p s-q+1), \quad q=1, \ldots, p, s \in\left[\frac{q-1}{p}, \frac{q}{p}\right) .
$$

Clearly, $t(1)=T$. The approximate control given by (3.1) in the new time horizon $[0,1]$ becomes

$$
\tilde{u}^{p}(s)=u^{p}(t(s))=\sum_{k=1}^{p} \sigma^{k} \chi_{\left[\frac{k-1}{p}, \frac{k}{p}\right)}(s),
$$

which has fixed switching times at $s=1 / p, \ldots,(p-1) / p$. Now, by using the time scaling transform (3.2)-(3.5), the dynamic system (2.1) is transformed into

$$
\dot{y}(s)=v^{p}(s) \tilde{f}(y(s), \sigma), \quad y(0)=x^{0}
$$

and the terminal condition (2.2) becomes

$$
y(1)=x^{f},
$$

where $y(s)=x(t(s))$ and $\tilde{f}(y(s), \sigma)=f\left(x(t(s)), \tilde{u}^{p}(s)\right)$. Similarly, applying the time scaling transform to the continuous state inequality constraints (2.3) yields

$$
g_{i}(y(s), \delta) \geq 0, \quad s \in[0, T], i=1, \ldots, N .
$$

To proceed further, let $y(\cdot \mid \sigma, \theta)$ denote the solution of System (3.8) corresponding to $(\sigma, \theta) \in \Xi \times \Theta$.

The approximation to Problem P may now be stated formally as follows.

Problem $\mathrm{P}(p)$. Given System (3.8), find a $(\sigma, \theta) \in \Xi \times \Theta$ such that the violation parameter $\delta$ is maximized subject to (3.5), (3.9) and (3.10).

To solve Problem $\mathrm{P}(p)$, we reformulate it as a problem of finding the largest root. First, we construct a new optimal control problem by applying the constraint transcription technique. Then a necessary condition and a sufficient condition are given showing the relationships between the new problem and the original problem. 
To derive an effective algorithm, a violation function dependent on $\delta$ is defined. The original problem is equivalent to the problem of finding the largest root of the violation function. The maximum violation parameter $\delta$ can be estimated by using the bisection search method. The optimal control software package MISER 3.3 [7] is used at each iteration.

To create an auxiliary optimal control problem, we begin by using the constraint transcription method [9], in which each of the continuous state inequality constraints is approximated by a smooth function $L_{i, \varepsilon}(y(s \mid \sigma, \theta), \delta)$, where

$$
L_{i, \varepsilon}(y(s \mid \sigma, \theta), \delta)=\left\{\begin{array}{cl}
-g_{i}(y(s), \delta) & \text { if } g_{i}(y(s), \delta)<-\varepsilon \\
\frac{\left(g_{i}(y(s), \delta)-\varepsilon\right)^{2}}{4 \varepsilon} & \text { if }-\varepsilon \leq g_{i}(y(s), \delta) \leq \varepsilon \\
0 & \text { if } g_{i}(y(s), \delta)>\varepsilon
\end{array}\right.
$$

We now define the auxiliary optimal control problem.

Problem $\mathrm{P}_{\delta}(p)$. For a fixed $\delta \in[0, \infty)$, determine $(\delta, \theta) \in \Xi \times \Theta$ to minimize the cost function

$$
J_{\varepsilon}(\sigma, \theta \mid \delta)=\int_{0}^{1} \sum_{i=1}^{N}\left(L_{i, \varepsilon}(y(s), \delta)\right) d s,
$$

over $\Xi \times \Theta$ subject to (3.5) and (3.9).

We give a necessary condition and a sufficient condition to relate Problem $\mathrm{P}(p)$ and Problem $\mathrm{P}_{\delta}(p)$.

REMARK 2. By Remark 1 we can show that $\int_{0}^{1} L_{i, \varepsilon}(y(s), \delta) d s$ is well defined for each $\delta \geq 0$.

REMARK 3. From (A2)-(A4) it is clear that for each $\delta, \partial g_{j}(y(s), \delta) / \partial y, j=$ $1, \ldots, N$, are continuous in $s \in[0,1]$, and $d y(s) / d s, i=1, \ldots, N$, is piecewise continuous in $s \in[0,1]$.

THEOREM 3.1. Let $\left(\sigma^{(0)}, \theta^{(0)}\right) \in \Xi \times \Theta$ and let $y^{(0)}$ be the corresponding solution of System (3.8) such that (3.9) is satisfied. Suppose that $\delta^{(0)}$ is such that (3.10) is satisfied. Then

$$
J_{\varepsilon}\left(\sigma^{(0)}, \theta^{(0)} \mid \delta^{(0)}\right)=\int_{0}^{1} \sum_{i=1}^{N} L_{i, \varepsilon}\left(y^{(0)}(s), \delta^{(0)}\right) d s \leq \frac{N \varepsilon}{4} .
$$

PROOF. By virtue of the constraint transcription defined in (3.11), it is clear that

$$
L_{i, \varepsilon}\left(y^{(0)}(s), \delta^{(0)}\right) \leq \frac{\varepsilon}{4}, \quad \forall s \in[0,1], i=1, \ldots, N
$$

Thus, the conclusion follows readily from (3.12). 
Before deriving the sufficient condition, the following lemma [6, Lemma 1] is quoted without proof.

LEMMA 3.2 ([6, Lemma 1]). Let $f$ be a nonnegative-valued function defined on $[0, T]$. If $f$ is continuously differentiable on $(0, T)$, then

$$
\int_{0}^{T} f(t) d t \geq \frac{\widehat{f}}{2} \min \left\{\frac{\widehat{f}}{M}, T\right\}
$$

where

$$
M=\max _{t \in[0, T]}\left|\frac{d f(t)}{d t}\right| \text { and } \widehat{f}=\max _{t \in[0, T]} f(t) .
$$

With the help of Lemma 3.2, we can derive the desired sufficient condition as in [6].

THEOREM 3.3. Let $\left(\sigma^{(0)}, \theta^{(0)}\right) \in \Xi \times \Theta$ such that (3.5) is satisfied and let $y^{(0)}$ be the corresponding solution of System (3.8) such that (3.9) is satisfied. Suppose that $\delta^{(0)}$ is such that

$$
J_{\varepsilon}\left(\sigma^{(0)}, \theta^{(0)} \mid \delta^{(0)}\right) \leq \frac{\varepsilon}{8} \min \left\{\frac{\varepsilon}{4 M}, 1\right\}
$$

where

$$
M=\max \left\{\left|\frac{\partial g_{i}\left(y^{(0)}(s), \delta^{(0)}\right)}{\partial y^{(0)}} \frac{d y^{(0)}(s)}{d s}\right|: s \in[0,1], i=1, \ldots, N\right\} .
$$

Then the constraints (3.10) are satisfied.

Proof. Suppose that there exists an $i, 0 \leq i \leq N$, such that the constraint (3.10) is not satisfied. Then, by virtue of (A2), there exists an open set $\vartheta_{i}$ with positive measure such that

$$
g_{i}\left(y^{(0)}(s), \delta^{(0)}\right)<0, \quad \forall s \in \vartheta_{i}
$$

From Remark 3, there exists a positive constant $M$ satisfying (3.15). Then we define

$$
M^{\prime}=\max \left\{\left|\frac{\partial g_{i, \varepsilon}\left(y^{(0)}(s), \delta^{(0)}\right)}{\partial y} \frac{d y^{(0)}(s)}{d s}\right|: s \in[0,1], i=1, \ldots, N\right\} .
$$

Clearly,

$$
\left|\frac{\partial L_{i, \varepsilon}\left(y^{(0)}(s), \delta^{(0)}\right)}{\partial y} \frac{d y^{(0)}(s)}{d s}\right| \leq\left|\frac{d L_{i, \varepsilon}\left(y^{(0)}(s), \delta^{(0)}\right)}{d g_{j}}\right|\left|\frac{\partial g_{i}\left(y^{(0)}(s), \delta^{(0)}\right)}{\partial y} \frac{d y^{(0)}(s)}{d s}\right| .
$$

Since

$$
\left|\frac{d L_{i, \varepsilon}\left(y^{(0)}(s), \delta^{(0)}\right)}{d g_{j}}\right| \leq 1, \quad \forall s \in[0,1],
$$


it is clear that

$$
M^{\prime} \leq M
$$

Thus, by Lemma 3.2,

$$
J_{\varepsilon}\left(\sigma^{(0)}, \theta^{(0)} \mid \delta^{(0)}\right)=\int_{0}^{1} L_{i, \varepsilon}\left(y^{(0)}(s), \delta^{(0)}\right) d t \geq \frac{\widehat{L_{\varepsilon}}}{2} \min \left\{\frac{\widehat{L_{\varepsilon}}}{M^{\prime}}, 1\right\},
$$

where

$$
\widehat{L_{\varepsilon}}=\max _{s \in[0,1]} L_{i, \varepsilon}\left(y^{(0)}(s), \delta^{(0)}\right) .
$$

By (3.11) and (3.16), we obtain

$$
\widehat{L_{\varepsilon}}>\frac{\varepsilon}{4}
$$

Thus, it follows from (3.17)-(3.19) that

$$
J_{\varepsilon}\left(\sigma^{(0)}, \theta^{(0)}, \delta^{(0)}\right) \geq \frac{\widehat{L_{\varepsilon}}}{2} \min \left\{\frac{\widehat{L_{\varepsilon}}}{M^{\prime}}, 1\right\}>\frac{\varepsilon}{8} \min \left\{\frac{\varepsilon}{4 M^{\prime}}, 1\right\} \geq \frac{\varepsilon}{8} \min \left\{\frac{\varepsilon}{4 M}, 1\right\} .
$$

This, however, contradicts (3.14). This completes the proof.

Note that Problem $\mathrm{P}_{\delta}(p)$ is an optimal control problem in canonical form. To solve it as a nonlinear optimization problem by using the optimal control software MISER 3.3 (see [7]), we need the gradient formulae of the objective function (3.12), the constraint function $\Phi(y(1))=y(1)-x^{f}=0$ from (3.9) and the constraint function $\Phi$ from (3.5). The gradient of the constraint function $\Phi(\theta)=\sum_{i=1}^{p}\left(\theta_{i} / p\right)-$ $T$ is given by $\partial \Phi(\theta) / \partial \theta=[1 / p, \ldots, 1 / p]^{\top}$.

The other two gradient formulae are given below. Their proofs are similar to that given for Theorem 5.2.1 reported in [9].

THEOREM 3.4. For each $\delta>0$, the gradients of the cost function $J_{\varepsilon}(\sigma, \theta \mid \delta)$ with respect to $\sigma$ and $\theta$ are

$$
\begin{aligned}
& \frac{\partial J_{\varepsilon}(\sigma, \theta \mid \delta)}{\partial \sigma}=\int_{0}^{1} \frac{\partial H_{0}\left(s, y(s), \sigma, \lambda_{0}(s)\right)}{\partial \sigma} d s, \\
& \frac{\partial J_{\varepsilon}(\sigma, \theta \mid \delta)}{\partial \theta}=\int_{0}^{1} \frac{\partial H_{0}\left(s, y(s), \sigma, \lambda_{0}(s)\right)}{\partial \theta} d s,
\end{aligned}
$$

where $H_{0}(s, y(s), \sigma, \lambda(s))$ is the Hamiltonian function for the cost function (3.12) given by

$$
H_{0}(s, y(s), \sigma, \lambda(s))=\sum_{i=1}^{N} L_{i, \varepsilon}(y(s), \delta)+\lambda_{0}(s) v^{p}(s) \tilde{f}(y(s), \sigma, s),
$$


and $\lambda_{0}(s)$ is the solution of the co-state differential equation

$$
\left(\dot{\lambda}_{0}(s)\right)^{\top}=-\frac{\partial H_{0}\left(s, y(s), \sigma, \lambda_{0}(s)\right)}{\partial y}
$$

with the boundary condition $\left(\lambda_{0}(1)\right)^{\top}=0$.

Similarly, for the constraint function $\Phi(y(1 \mid \sigma, \theta))=y(1 \mid \sigma, \theta)-x^{f}$, we have the following theorem.

THEOREM 3.5. For each $\delta$, the gradients of the constraint function $\Phi(y(1 \mid \sigma, \theta))$ with respect to $\sigma$ and $\theta$ are

$$
\begin{aligned}
& \frac{\partial \Phi(y(1 \mid \sigma, \theta))}{\partial \sigma}=\int_{0}^{1} \frac{\partial H_{1}\left(s, y(s), \sigma, \lambda_{1}(s)\right)}{\partial \sigma} d s, \\
& \frac{\partial \Phi(y(1 \mid \sigma, \theta))}{\partial \theta}=\int_{0}^{1} \frac{\partial H_{1}\left(s, y(s), \sigma, \lambda_{1}(s)\right)}{\partial \theta} d s,
\end{aligned}
$$

where $H_{1}(s, y(s), \sigma, \lambda(s))$ is the Hamiltonian function for the constraint function (3.9) given by

$$
H_{1}(s, y(s), \sigma, \lambda(s))=\lambda_{1}(s) v^{p}(s) \tilde{f}(y(s), \sigma, s),
$$

and $\lambda_{1}(s)$ is the solution of the co-state differential equation

$$
\left(\dot{\lambda}_{1}(s)\right)^{\top}=-\frac{\partial H_{1}\left(s, y(s), \sigma, \lambda_{1}(s)\right)}{\partial y}
$$

with the boundary condition

$$
\left(\lambda_{1}(1)\right)^{\top}=\frac{\partial \phi(y(1))}{\partial y} .
$$

Let

$$
\Psi_{i}^{p}(\delta)=\max _{0 \leq s \leq 1}\left\{\max \left(-g_{i}(\widehat{y}(s), \delta), 0\right)\right\}, \quad i=1, \ldots, N,
$$

where $\widehat{y}(s)$ is the solution obtained by solving Problem $\mathrm{P}_{\delta}(p)$, and define the violation function

$$
\Psi^{p}(\delta)=\max _{0 \leq i \leq N} \Psi_{i}^{p}(\delta) .
$$

Clearly, $\Psi^{p}(\delta)=0$ if and only if (3.10) is satisfied. Using (A2), we can show that the real-valued function $\Psi^{p}(\delta)$ is a nondecreasing function of $\delta$. By (A3), there exists a $\delta$ such that $\Psi^{p}(\delta)=0$. The maximum value of $\delta$ such that $\Psi^{p}(\delta)=0$ is the largest zero of the real-valued function $\Psi^{p}(\delta)$ in $[0,+\infty)$.

We now see that Problem $\mathrm{P}(p)$ is equivalent to the problem of finding the largest zero of $\Psi^{p}(\delta)$. We shall use a zero-finding algorithm to generate a sequence of points $\delta_{k}, k=1,2, \ldots$, which converges to $\delta^{*}$, where $\delta^{*}$ is the largest zero of $\Psi^{p}(\delta)$. For each $\delta_{k}$ we evaluate $\Psi^{p}\left(\delta_{k}\right)$ after solving the optimal control problem $\mathrm{P}_{\delta_{k}}(p)$. 
Then, for a fixed integer $p$, we present Algorithm 1, the bisection search method to search for the largest zero $\delta^{p, *}$ of the function $\Psi^{p}$. Given two starting values $\delta_{1}$ and $\delta_{2}$, the recursive formula for the bisection search method is

$$
\delta_{k+1}=\frac{1}{2}\left(\delta_{k}+\delta_{k-1}\right), \quad k=2,3, \ldots .
$$

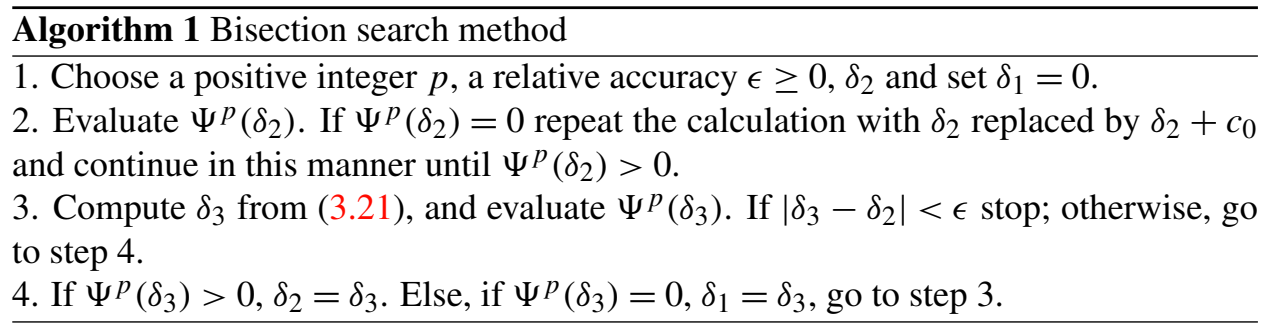

REMARK 4. In Step 2 of Algorithm 1, $c_{0}$ is chosen so as to generate a point $\delta_{2}$ such that $\Psi^{p}\left(\delta_{2}\right)>0$.

REMARK 5. $\Psi^{p}(\delta)$ is calculated by using formula (3.20) after solving Problem $\mathrm{P}_{\delta_{k}}(p)$.

REMARK 6. The interval of uncertainty for this bisection search method is $(0.5)^{k}$ of the original interval.

REMARK 7. Let $\delta^{p, *}$ and $\delta^{*}$ be, respectively, the optimal solutions of Problem $\mathrm{P}(p)$ and Problem P. Then $\delta^{p, *} \rightarrow \delta^{*}$ as $p \rightarrow \infty$. The proof is similar to that given for [9, Theorem 8.5.2].

\section{Obstacle-avoidance problems}

In this section, we consider an autonomous mobile robot in the framework of behaviour-based control (see [1]). The robot is required to reach a pre-specified target from a given initial condition (position, orientation) while avoiding obstacles along the way.

The dynamical equations for the robot are

$$
\begin{gathered}
\left\{\begin{array}{l}
\dot{x}=v \cos \phi, \\
\dot{y}=v \sin \phi, \\
\dot{\phi}=\omega,
\end{array}\right. \\
|\omega(t)| \leq 1, \quad t \in[0, \infty)
\end{gathered}
$$

where $(x, y)$ is the position of the robot, $\phi$ is its orientation, and $v$ and $\omega$ are its translational and angular velocities. $v$ is assumed constant and $\omega$ is a control variable. In our problem, $v=1$. We will discuss situations with one and two obstacles. We define a circle with the obstacle as its centre and $\delta$ its radius.

4.1. One-obstacle avoidance problem We first solve the minimum time optimal control problem for a robot. 

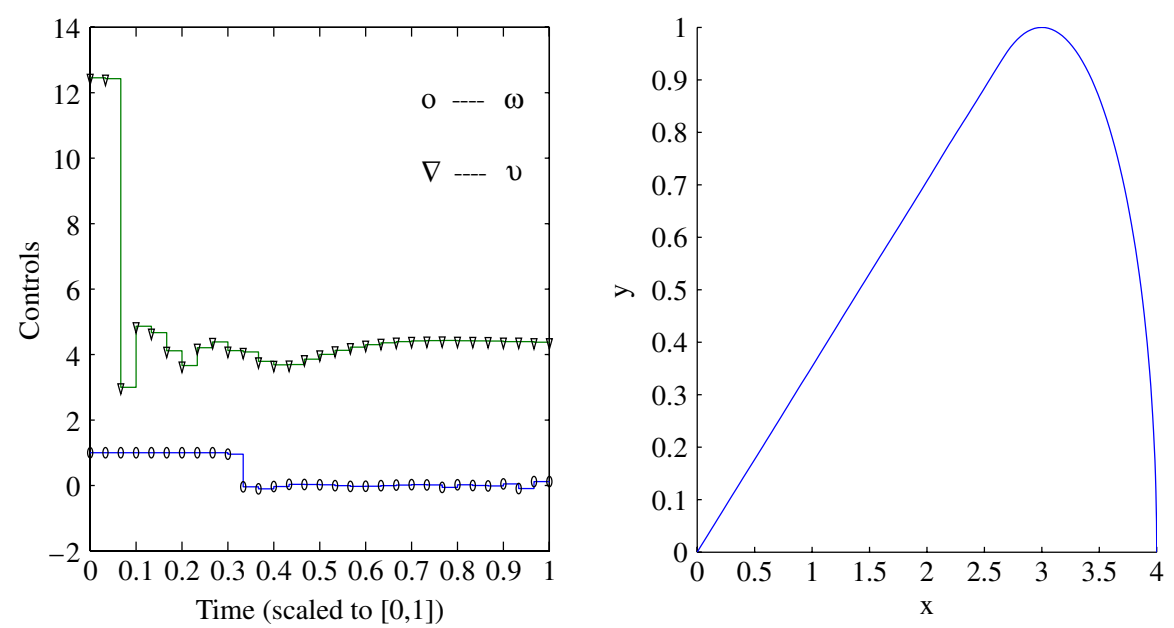

FIGURE 1. When an obstacle is not present: (left) optimal control and (right) the optimal trajectory of the robot.

Problem E. Consider a robot with dynamics given by (4.1), initial condition $(x(0), y(0), \phi(0))=(4,0, \pi / 2)$ and target condition $(x(T), y(T))=(0,0)$. Determine the minimum time required to reach the target.

We solve Problem E by using optimal control software package MISER 3.3. The optimal control $u^{*}$ and the optimal trajectory $x^{*}$ are depicted in Figure 1. The minimum time to reach the target is $T^{*}=4.7391$.

Suppose now that an obstacle is discovered at the location

$$
\bar{x}=[1.78682,0.63301]^{\top},
$$

which is a point on the optimal path. Suppose that we are allowed to have a small amount of extra time, say $5 \%$ more than the minimum time $T^{*}$, for the robot to reach the target. With this extra allowance, how do we steer the trajectory of the robot in such a way that the minimum distance between the trajectory and the obstacle is maximized? This task is equivalent to finding a control $u$ such that $\delta$ is maximized subject to the nonlinear inequality constraint

$$
(x(t)-1.78682)^{2}+(y(t)-0.63301)^{2} \geq \delta^{2}, \quad \forall t \in[0, T],
$$

and the time constraint

$$
T \leq(1+0.05) T^{*} \text {. }
$$

After applying the control parametrization and the time scaling transform outlined in Section 3, the time constraint (4.2) becomes a constraint on the control parameters given below.

We solve this problem by using Algorithm 1. The maximum value of $\delta=0.49539$ is obtained. The corresponding optimal trajectory is shown in Figure 2. 

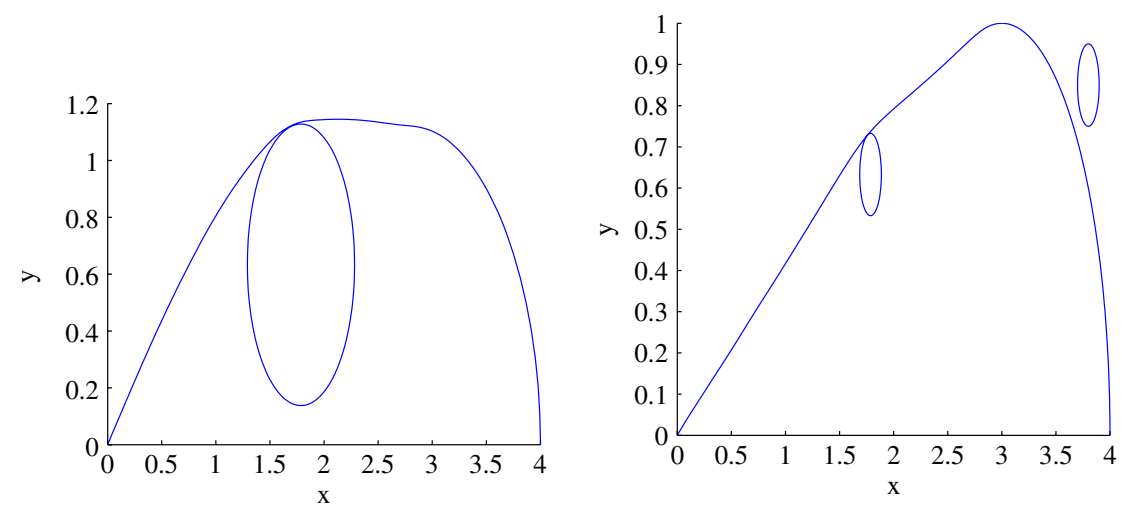

FIGURE 2. The optimal trajectory with (left) one obstacle and (right) two obstacles.

4.2. Two-obstacle avoidance problem Suppose now there are two obstacles. One is located at $(1.78682,0.63301)^{\top}$ and the other is located at $(3.80000,0.85000)^{\top}$. These are points on the optimal path. Allowing the time to reach the target to be increased to less than or equal to $(1+\alpha) T^{*}$, that is,

$$
\sum_{i=1}^{p} \frac{\theta_{i}}{p}=T \leq(1+\alpha) T^{*},
$$

with $\alpha=5 \%$, we use Algorithm 1 to solve the corresponding maximum two-obstacle avoidance problem. The maximum value of $\delta$ obtained is 0.0996 . The corresponding optimal trajectory is shown in Figure 2.

\section{Abort landing of an aircraft in a windshear downburst}

In this section, we consider the problem of the abort landing of a Boeing 727 passenger aircraft in the presence of a windshear downburst as considered in $[4,8]$.

To set up the equations of motion, we assume that the aircraft is a particle of constant mass, the flight takes place in a vertical plane, and Newton's second law is valid in a system with the Earth taken as fixed. Moreover, the wind flow field is assumed to be steady. Under these assumptions, the dynamical equations are

$$
\left\{\begin{aligned}
\dot{x}= & V \cos \gamma+W_{x}, \quad \dot{h}=V \sin \gamma+W_{h}, \\
\dot{V}= & \frac{T}{m} \cos (\alpha+\delta)-\frac{D}{m}-g \sin \gamma-\left(\dot{W}_{x} \cos \gamma+\dot{W}_{h} \sin \gamma\right), \\
\dot{\gamma}= & \frac{T}{m V} \sin (\alpha+\delta)+\frac{L}{m V}-\left(\frac{1}{V}\right) g \cos \gamma \\
& +\left(\frac{1}{V}\right)\left(\dot{W}_{x} \sin \gamma-\dot{W}_{h} \cos \gamma\right) .
\end{aligned}\right.
$$

The state variables are the horizontal distance $x$, the altitude $h$, the relative velocity $V$, and the relative path inclination $\gamma$. In the formulation above, $\alpha$, the relative angle of 

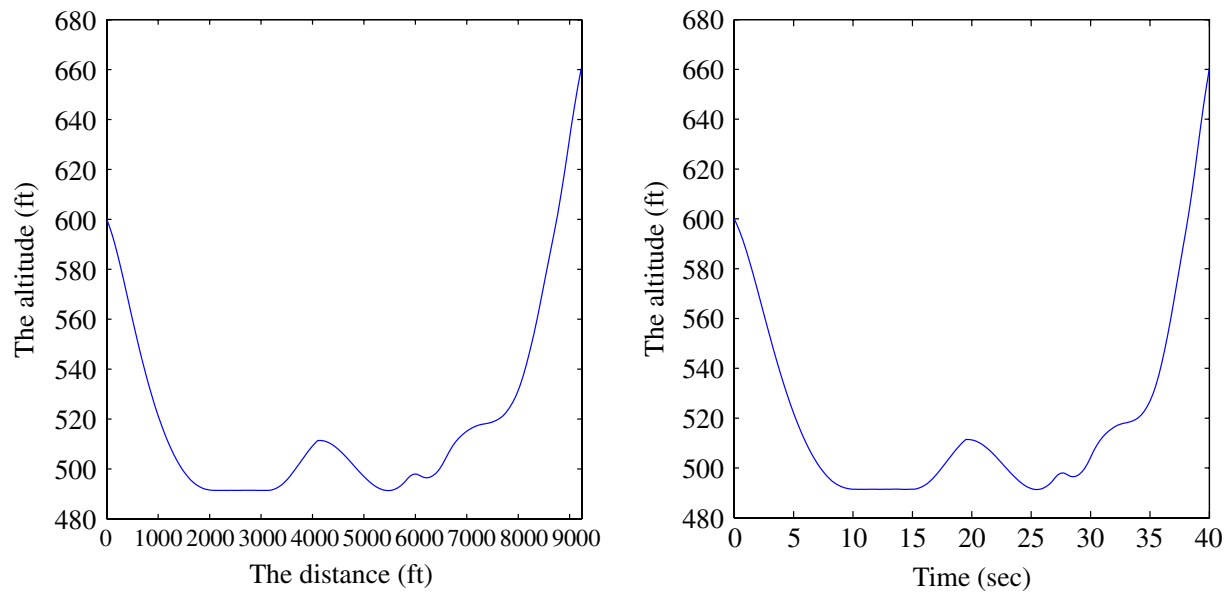

FIGURE 3. Altitude versus distance (left) and time (right).

attack of the airplane wing with respect to the wind velocity, is chosen as the control variable.

The approximation of the aerodynamic forces acting on the airplane is given by

$$
\left\{\begin{array}{l}
T=\beta T_{*}, \quad T_{*}=A_{0}+A_{1} V+A_{2} V^{2}, \quad D=\frac{C_{D} \rho S V^{2}}{2}, \\
C_{D}(\alpha)=B_{0}+B_{1} \alpha+B_{2} \alpha^{2}, \quad L=\frac{C_{L} \rho S V^{2}}{2}, \\
C_{L}(\alpha)= \begin{cases}C_{0}+C_{1} \alpha, & \alpha \leq \alpha_{*}, \\
C_{0}+C_{1} \alpha+C_{2}\left(\alpha-\alpha_{*}\right)^{2}, & \alpha_{*} \leq \alpha \leq \alpha_{\max } .\end{cases}
\end{array}\right.
$$

Here, $T, D$ and $L$ denote the thrust, drag and lift, respectively. The power setting $\beta$ is specified in advance:

$$
\beta(t)= \begin{cases}\beta_{0}+\dot{\beta}_{0} t, & 0 \leq t \leq t_{0}, \\ 1, & t_{0}<t \leq t_{f} .\end{cases}
$$

The windshear model, which is valid for $h \leq 1000 \mathrm{ft}$, is given by

$$
W_{x}=k A(x), \quad W_{h}=k\left(h / h_{*}\right) B(x),
$$

with

$$
A(x)= \begin{cases}-50+a x^{3}+b x^{4}, & 0 \leq x \leq 500 \\ \frac{x-2300}{40}, & 500<x \leq 4100 \\ 50-a(4600-x)^{3}-b(4600-x)^{4}, & 4100<x \leq 4600 \\ 50, & 4600<x\end{cases}
$$


TABLE 1. Model data for a Boeing 727 aircraft.

\begin{tabular}{lll}
\hline Equations (5.1), (5.2) & Equations (5.2), (5.3) & Equation (5.2) \\
\hline$\rho=0.2203 \times 10^{-2} \mathrm{lb} \mathrm{sec}^{2} \mathrm{ft}^{-4}$ & $A_{0}=44560 \mathrm{lb}$ & $B_{0}=0.1551$ \\
$S=0.1560 \times 10^{4} \mathrm{ft}^{2}$ & $A_{1}=-23.98 \mathrm{lb} \mathrm{sec} \mathrm{ft}{ }^{-1}$ & $B_{1}=0.12369 \mathrm{rad}^{-1}$ \\
$g=3.2171 \times 10^{1} \mathrm{ft} \mathrm{sec}^{-2}$ & $A_{2}=0.01442 \mathrm{lb} \mathrm{sec}^{2} \mathrm{ft}^{-2}$ & $B_{2}=2.4203 \mathrm{rad}^{-2}$ \\
$m g=150000 \mathrm{lb}$ & $\beta^{0}=0.3825$ & $C_{0}=0.7125$ \\
$\delta=2 \mathrm{deg}$ & $\dot{\beta}_{0}=0.2 \mathrm{sec}^{-1}$ & $C_{1}=6.0877 \mathrm{rad}^{-1}$ \\
& $t_{0}=\left(1-\beta_{0}\right) / \dot{\beta}_{0}$ & $C_{2}=-9.0277 \mathrm{rad}^{-2}$ \\
& $t_{f}=40 \mathrm{sec}$ & $\alpha_{*}=12 \mathrm{deg}$ \\
\hline Equations $(5.4)-(5.6)$ & $\alpha_{\max }=17.2 \mathrm{deg}$ & \\
\hline$k=1$ & Equation $(5.7)$ & \\
$h_{*}=1000 \mathrm{ft}$ & $x_{0}=0 \mathrm{ft}$ & \\
$a=6 \times 10^{-8} \mathrm{sec}^{-1} \mathrm{ft}^{-2}$ & $\gamma_{0}=-2.249 \mathrm{deg}$ & \\
$b=-4 \times 10^{-11} \mathrm{sec}^{-1} \mathrm{ft}^{-3}$ & $h_{0}=600 \mathrm{ft}$ & \\
$c=-\ln (25 / 30.6) \times 10^{-12} \mathrm{ft}^{-4}$ & $\alpha_{0}=7.353 \mathrm{deg}$ & \\
$d=-8.02881 \times 10^{-8} \mathrm{sec}^{-1} \mathrm{ft}^{-2}$ & $V_{0}=239.7 \mathrm{ft} \mathrm{sec}$ & \\
$e=6.28083 \times 10^{-11} \mathrm{sec}^{-1} \mathrm{ft}^{-3}$ & & \\
\hline
\end{tabular}

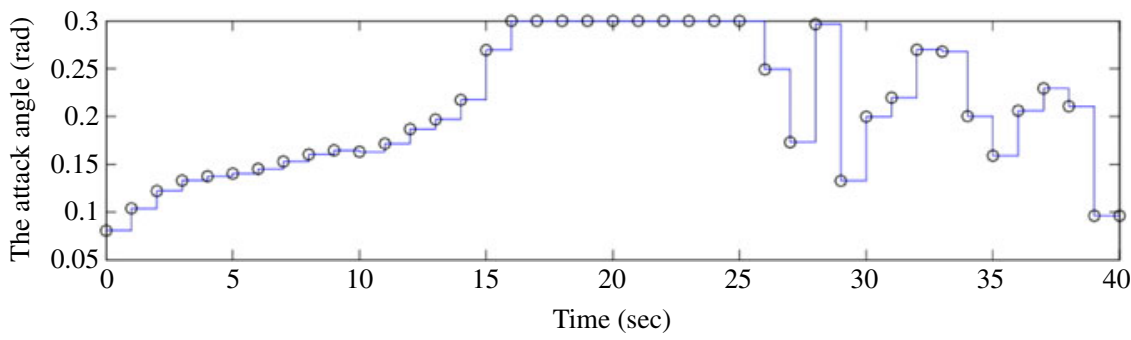

FIGURE 4. Relative angle of attack.

and

$$
B(x)= \begin{cases}d x^{3}+e x^{4}, & 0 \leq x \leq 500 \\ -51 \exp \left[-c(x-2300)^{4}\right], & 500 \leq x \leq 4100 \\ d(4600-x)^{3}-e(4600-x)^{4}, & 4100 \leq x \leq 4600 \\ 0, & 4600 \leq x\end{cases}
$$

The angle of attack $\alpha$, is subject to the inequality constraint $\alpha \leq \alpha_{\max }$. The initial conditions are

$$
x(0)=x_{0}, \quad h(0)=h_{0}, \quad V(0)=V_{0}, \quad \gamma(0)=\gamma_{0}, \quad \alpha(0)=\alpha_{0},
$$

and the terminal condition is $\gamma\left(t_{f}\right)=\gamma_{f}$. All the required data are given in Table 1 . 


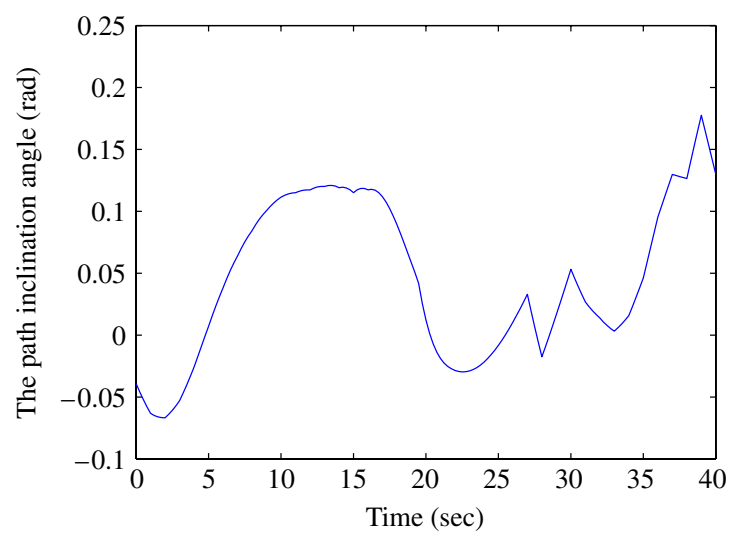

FIGURE 5. Relative path inclination angle.

To reduce the risk of crashing into the ground, the minimal altitude is required to be maximized, that is,

$$
\max _{\alpha \leq \alpha_{\max }} \min _{0 \leq t \leq t_{f}} h(t) .
$$

This is equivalent to maximizing $\delta$ subject to the continuous state inequality constraint $g(h(t), \delta)=h(t)-\delta \geq 0$.

The problem is now a special case of Problem P. The computation method developed in Section 3 is used to solve it. The minimal altitude is $492 \mathrm{ft}$, and the trajectories of the altitude $h$, the relative attack angle $\alpha$ and the relative path inclination angle $\gamma$ are shown in Figures 3-5, respectively. The minimal altitude is slightly larger than that reported in [5]. Our method does not require a good initial guess and the optimization process converges very rapidly.

\section{Acknowledgements}

This work was supported by a grant from the Australia Research Council, Major Program of National Natural Science Foundation of China (No. 60710002), and the China Scholarship Council.

\section{References}

[1] R. Arkin, Behavior-based robotics (MIT Press, Cambridge, MA, 1998).

[2] H. Axelsson, M. Egerstedt and Y. Wardi, "Reactive robot navigation using optimal timing control", Proc. of the American Control Conference (2005) 4929-4934.

[3] M. Boccadoro, Y. Wardi, M. Egerstedt and E. Verriest, "Optimal control of switching surfaces in hybrid dynamical systems", Discrete Event Dyn. Syst. 15 (2005) 433-448.

[4] R. Bulirsch, F. Montrone and H. J. Pesch, "Abort landing in the presence of windshear as a minmax optimal control problem, Part 1: Necessary conditions", J. Optim. Theory Appl. 70 (1991) 1-23. 
[5] R. Bulirsch, F. Montrone and H. J. Pesch, "Abort landing in the presence of windshear as a minmax optimal control problem, Part 2: Multiple shooting and homotopy", J. Optim. Theory Appl. 70 (1991) 223-254.

[6] C. J. Goh and K. L. Teo, "Alternative algorithms for solving nonlinear function and functional inequalities", Appl. Math. Comput. 41 (1991) 159-177.

[7] L. S. Jennings, M. E. Fisher, K. L. Teo and C. J. Goh, "Miser3, Optimal control software version 3: Theory and user manual" Centre for Applied Dynamics and Optimization, The University of Western Australia, URL: http://www.cado.uwa.edu.au/miser/manual.html, 2004.

[8] A. Miele, "Aircraft survival in windshear flight", in: Mechanics and control, Volume 151 of Lecture Notes in Control and Information Sciences (eds J. M. Skowronski, H. Flashner and R. S. Guttalu) (Springer-Verlag, Berlin, 1991) 281-294.

[9] K. L. Teo, C. J. Goh and K. H. Wong, A unified computational approach for optimal control problems (Longman Scientific and Technical, New York, 1991).

[10] K. L. Teo, L. S. Jennings, H. W. J. Lee and V. L. Rehbock, "The control parameterization enhancing transformation for constrained optimal control problems", J. Aust. Math. Soc. Ser. B 40 (1997) 314-335.

[11] K. L. Teo and X. Q. Yang, "A root finding approach for continuous minmax optimal control problems", Nonlinear Stud. 4 (1997) 37-52. 